\title{
Multilocus Genetic Profile for Dopamine Signaling Predicts Ventral Striatum Reactivity
}

\author{
Yuliya S Nikolova', Robert E Ferrell ${ }^{2}$, Stephen B Manuck ${ }^{3}$ and Ahmad R Hariri*, 1,4 \\ 'Department of Psychology and Neuroscience, Duke University, Durham, NC, USA; 'Department of Human Genetics, University of Pittsburgh, \\ Pittsburgh, PA, USA; ${ }^{3}$ Department of Psychology, University of Pittsburgh, Pittsburgh, PA, USA; ${ }^{4}$ Institute for Genome Sciences and Policy, Duke \\ University, Durham, NC, USA
}

\begin{abstract}
Research integrating neuroimaging and molecular genetics has yielded important insights into how variability in brain chemistry predicts individual differences in brain function, behavior and related risk for psychopathology. However, existing studies have been limited by their focus on the independent effects of single polymorphisms with modest impact on brain chemistry. Here, we explored the effects of five functional polymorphisms affecting dopamine (DA) signaling on reward-related ventral striatum (VS) reactivity, measured with BOLD PMRI, in a sample of 69 Caucasians. We also compiled individual multilocus genetic profile scores reflecting the additive effects of alleles conferring relatively increased DA signaling across the five polymorphic loci: DATI 9-repeat, DRD4 7-repeat, DRD2 - I4IC Del, DRD2 Taq IA C (A2), and COMT ${ }^{158}$ Met. These multilocus DA profile scores accounted for $10.9 \%$ of the inter-individual variability in reward-related VS reactivity. In contrast, none of the individual polymorphisms accounted for significant variability. Our results show that biologically informed multilocus genetic profiles have unique promise as indices of variability in brain chemistry that may yield advances in mapping individual differences in behaviorally relevant brain function. In turn, such genetic profiles may fuel gene-environment interactions research establishing trajectories of risk for psychopathology.

Neuropsychopharmacology (20II) 36, 1940-1947; doi:I0.1038/npp.20I I.82; published online I8 May 20I I
\end{abstract}

Keywords: genetic profile; dopamine; ventral striatum; reward; $\mathrm{PMRI}$

\section{INTRODUCTION}

Two rapidly emerging and highly complementary strategies have accelerated progress into biological mechanisms mediating individual differences in behavior and related risk for psychopathology: imaging genetics and geneenvironment interactions research. Through the systematic mapping of common genetic polymorphisms affecting brain chemistry onto variability in brain structure and function, imaging genetics has established multiple fundamental mechanisms through which individual differences in behavior emerge and bias responses to the environment (Hariri, 2009). In parallel, gene-environment interactions research has demonstrated how such genetically mediated variability in behaviorally relevant brain function translates into individual risk for psychopathology upon exposure to environmental stress or adversity (Caspi and Moffitt, 2006).

* Correspondence: Dr AR Hariri, Department of Psychology and Neuroscience, Box 90086, 417 Chapel Drive, Durham, NC 277080086, USA, Tel: + | 91968| 1039, Fax: +| 9196605726 ,

E-mail: ahmad.hariri@duke.edu

Received 4 February 2011; revised 25 March 201।; accepted I5 April 2011
Imaging genetics studies to date, however, have been almost universally limited by their reliance on single genetic loci to model variability in complex brain chemistry and, subsequently, brain function. Recent studies have begun to recognize the importance of considering the simultaneous involvement of multiple genes in the regulation of these pathways by taking into account epistatic interactions among polymorphic loci (Buckholtz et al, 2007; Nicodemus et al, 2010; Pezawas et al, 2008). Nonetheless, studies of this kind have typically focused on no more than two genes/polymorphisms at a time and those which have taken more into account have done so within the framework of a data-driven approach (Nicodemus et al, 2010; Potkin et al, 2009; Seshadri et al, 2007). As multiple functional polymorphisms of various effect sizes are likely to shape overall variability in brain function, one strategy for extending and expanding the utility of this research is to establish biologically founded multilocus genetic profiles that represent the cumulative effect of multiple polymorphic loci of known functionality on a specific signaling mechanism (Plomin et al, 2009). Individual polymorphic loci account for a small proportion of phenotypic variance such that their independent effects are unlikely to produce statistically significant effects especially in relatively small 
samples. The simultaneous consideration of multiple functional loci through a multilocus genetic profile score may allow for the inclusion of polymorphisms with nonsignificant independent effects, which only collectively account for significant proportions of variability. In turn, such genetic profiles may serve as the foundation for geneenvironment interactions research that can establish trajectories of risk for psychopathology applicable at the level of the individual.

In this study, we sought to establish the utility of multilocus genetic profiles representing the cumulative biological impact of multiple functional polymorphic loci in mapping individual differences in brain function. The simultaneous consideration of multiple polymorphisms has already been successfully used to explain variability in antidepressant treatment response (Ising et al, 2009) and to model individual differences in sensation seeking (Derringer et al, 2010) and basal ganglia response to reward (Dillon et al, 2010). However, no study to date has created a biologically informed multilocus genetic profile representing variability in neurotransmitter signaling across multiple genes that can be used to explain individual differences in behaviorally relevant brain function. The neural target of our study was variability in the responsiveness of the ventral striatum (VS), a central node of a distributed corticostriatal circuitry supporting reward-related and appetitive behaviors (Gan et al, 2010; Tanaka et al, 2004), which is also implicated in the pathophysiology of mood, impulse, and substance use disorders (Buckholtz et al, 2010a, b; Dalley et al, 2007). The genetic target of our study was dopamine (DA), which has a key role in modulating the responsiveness of the VS (Sesack and Grace, 2010). We hypothesized that multilocus genetic profile scores representing relatively increased DA signaling, would significantly predict increased VS reactivity, and that the variance in reactivity explained by the profile scores would be significantly greater than that associated with any single locus.

All five loci included in the genetic profile were carefully selected based on their previous links with functional changes in DA transmission and/or VS reactivity. The DAT1 9-repeat allele of a 40-base pair (bp) variable number tandem repeat (VNTR) within the $3^{\prime}$ untranslated region ( $3^{\prime}$ UTR) of the DA transporter gene (SLC6A3) has been linked to reduced DA reuptake and increased striatal DA signaling (Heinz et al, 2000; VanNess et al, 2005). Similarly, the deletion allele of an insertion/deletion polymorphism (DRD2 -141C Ins/Del; rs1799732) within the promoter region of the DA receptor D2 gene (DRD2) has been associated with reduced expression of DRD2 (Arinami et al, 1997), and has been implicated in increased VS reactivity (Forbes et al, 2009). We also considered the DRD2 Taq1A polymorphism, a C/T SNP (rs1800497) located in the ankyrin repeat and kinase-domain containing 1 (ANKK1) gene. Relative to the $\mathrm{T}$ (A1) allele, the $\mathrm{C}$ (A2) allele has been associated with increased DA signaling (Noble et al, 1991), increased striatal glucose metabolism (Noble et al, 1997) and reactivity to reward (Stice et al, 2008). The fourth polymorphism we considered was a 48-bp VNTR within the $\mathrm{DA}$ receptor $\mathrm{D} 4$ gene $(D R D 4)$. The 7-repeat allele of this VNTR has been previously linked to reduced DRD4mediated postsynaptic inhibition and hence increased DA signaling (Wang et al, 2004) as well as increased VS reactivity (Forbes et al, 2009). Finally, our genetic profile score incorporated a functional SNP (rs4680) within the third exon of the catechol-O-methyltransferase gene (COMT), which results in nonsynonymous $\mathrm{Val} / \mathrm{Met}$ substitution (COMT Val $\left.{ }^{158} \mathrm{Met}\right)$. The Met allele has been associated with decreased enzymatic degradation of DA (Chen et al, 2004) and increased VS reactivity (Dreher et al, 2009; Yacubian et al, 2007).

\section{PARTICIPANTS AND METHODS}

\section{Participants}

A total of 103 subjects were recruited from a parent study, the Adult Health and Behavior project, which assessed a wide range of behavioral and biological traits among nonpatient, middle-aged, community volunteers. All participants provided informed consent in accord with local guidelines, and were in good general health. The participants were free of the following study exclusions: (1) medical diagnoses of cancer, stroke, diabetes requiring insulin treatment, chronic kidney or liver disease, or lifetime history of psychotic symptoms; (2) use of psychotropic, glucocorticoid, or hypolipidemic medication; (3) conditions affecting cerebral blood flow and metabolism (eg, hypertension); and (4) diagnosis of any current DSM-IV axis I disorder (First et al, 1996). Given the general confounds of population stratification, we limited our analyses to 69 Caucasian subjects (37 women; mean age $44.46 \pm 6.66$ years) with overlapping reward-related VS data and genotypes at all five loci of interest.

\section{Genetic Profile Scores}

We compiled individual genetic profile scores reflecting the total number of variants that have each been previously associated with relatively increased striatal DA signaling and/or VS reactivity across five functional polymorphic loci: SLC6A3 3' 40-bp VNTR (DAT1), DRD2 -141C Ins/Del (rs1799732), DRD2 Taq1A (rs1800497), DRD4 exon 3 48-bp VNTR, and COMT Val ${ }^{158}$ Met (rs4680) (for genotyping, see Supplementary Methods). Across all loci, relatively 'high' DA genotypes were assigned a score of 1, 'low' DA genotypes a score of 0 , and 'intermediate' DA genotypes a score of 0.5 . These scores at each locus were then totaled to create an individual profile score (Table 1).

Consistent with previous research suggesting a dominant role for the 9-repeat allele (Heinz et al, 2000; van de Giessen et al, 2009; VanNess et al, 2005), DAT1 9-repeat allele carriers were coded as having a 'high' DA genotype, while 10-repeat allele homozygotes were coded as having a 'low' DA genotype. Drawing on previous reports, we also established two genotype groups for the DRD2 -141C locus: -141C deletion carriers (Ins/Del) and non-carriers (Ins/Ins), and designated -141C Del carriers as the 'high' and non-carriers as 'low' DA genotypes. Since previous studies of DRD2 Taq1A have used either T (A1) or C (A2) allele homozygotes as a reference group (Bakker et al, 2008; Jonsson et al, 1999; Kwon et al, 2008; Pohjalainen et al, 1998), and other research suggests additive effects for the number of DRD2 Taq1A alleles on relative change in DRD2 
Table I Composition and Distribution of Multilocus Genetic Profile Scores

\begin{tabular}{llll}
\hline Polymorphism & Genotypes & N & DA profile score \\
\hline DRD2 - I4IC Ins/Del & Del carrier & 14 & High \\
& Ins/Ins & 55 & Low \\
DATI VNTR & 9 -repeat carrier & 35 & High \\
& I0/I0 & 34 & Low \\
DRD4 VNTR & 7-repeat carrier & 42 & High \\
& All others & 27 & Low \\
COMT Val ${ }^{158}$ Met & Met/Met & 12 & High \\
& Val/Met & 41 & Intermediate \\
& Val/Val & 16 & Low \\
DRD2 Taq IA & C/C & 43 & High \\
& C/T & 23 & Intermediate \\
& T/T & 3 & Low \\
\hline
\end{tabular}

Individual genetic profile scores represent the sum of 'high' DA genotypes across five functional polymorphic loci. 'High' genotypes received a score of I, 'low' genotypes a score of 0 , and 'intermediate' genotypes a score of 0.5 . For example, the genetic profile score for an individual with the following five polymorphisms-DATI 9repeat carrier, DRD4 7-repeat carrier, DRD2 TaqIA T homozygote, DRD2 -I4IC Del carrier, and COMT heterozygote-is $3.5(I+1+0+1+0.5)$.

expression levels (Noble et al, 1991), we modeled allele load effects of the DRD2 Taq1A on overall DA transmission with C allele homozygotes designated as the 'high' DA genotype, T allele homozygotes as the 'low' DA genotype and heterozygotes as 'intermediate' DA genotype. The DRD4 7-repeat allele carriers were considered 'high', while other allele combinations were considered 'low' DA genotypes. Finally, consistent with additive effects of the Met allele of COMT Val ${ }^{158}$ Met (Chen et al, 2004; Egan et al, 2001), we established three genotype groups in relation to this locus: Val homozygotes, Val/Met heterozygotes, and Met homozygotes. For the purposes of the DA profile scores, Met allele homozygotes were considered 'high', Val allele homozygotes 'low', and heterozygotes 'intermediate' genotypes.

\section{VS Reactivity Paradigm}

As described previously (Forbes et al, 2009; Gianaros et al, 2010; Hariri et al, 2006; Hariri et al, 2009), our blockeddesign paradigm consisted of pseudorandom presentation of trials wherein participants played a card guessing game and received positive or negative feedback (ie, correct or incorrect guess) for each trial. Our task was selected primarily with the aim of robustly engaging the VS, so that individual differences in VS responsiveness could be recorded and mapped onto genetic background. Participants were told that their performance on the card game would determine a monetary reward to be received at the end of the game. During each trial, participants had $3 \mathrm{~s}$ to guess, via button press, whether the value of a visually presented card was higher or lower than 5 (index and middle finger, respectively). After a choice was made, the numerical value of the card was presented for $500 \mathrm{~ms}$ and followed by appropriate feedback (green upward-facing arrow for positive feedback; red downward-facing arrow for negative feedback) for an additional $500 \mathrm{~ms}$. A crosshair was then presented for $3 \mathrm{~s}$, for a total trial length of $7 \mathrm{~s}$. Each block comprises five trials, with three blocks each of predominantly positive feedback ( $80 \%$ correct) and three of predominantly negative feedback (20\% correct) interleaved with three control blocks. During control blocks, participants were instructed to simply make alternating button presses during the presentation of an ' $x$ ' (3s) which was followed by an asterisk $(500 \mathrm{~ms})$ and a yellow circle $(500 \mathrm{~ms})$. Each block was preceded by an instruction of 'Guess Number' (positive or negative feedback blocks) or 'Press Button' (control blocks) for $2 \mathrm{~s}$ resulting in a total block length of $38 \mathrm{~s}$ and a total task length of $342 \mathrm{~s}$. Participants were unaware of the fixed outcome probabilities associated with each block and were led to believe that their performance would determine a net monetary gain at the end of the scanning session. Instead, all participants received $\$ 10$. We included one incongruent trial within each task block (eg, one of five trials during positive feedback blocks was incorrect resulting in negative feedback) to prevent participants from anticipating the feedback for each trial and to maintain participants' engagement and motivation to perform well (for BOLD Acquisition Parameters, see Supplementary Methods).

\section{BOLD fMRI Data Analysis}

Whole-brain image analysis was completed using SPM2 (http://www.fil.ion.ucl.ac.uk/spm). Images for each participant were realigned to the first volume in the time series to correct for head motion. Data sets were then selected for their high quality (scan stability) as demonstrated by small $\left(\leqslant 2 \mathrm{~mm}\right.$ and $2^{\circ}$ ) motion correction. On the basis of this criterion, data from all 69 participants were included in subsequent analyses. Realigned images were spatially normalized into a standard stereotactic space (Montreal Neurological Institute template) using a 12-parameter affine model. These normalized images were then smoothed to minimize noise and residual differences in gyral anatomy with a Gaussian filter, set at $6 \mathrm{~mm}$ full-width at half-maximum. Voxel-wise signal intensities were ratio normalized to the whole-brain global mean. Following preprocessing, linear contrasts employing canonical hemodynamic response functions were used to estimate differential effects of feedback (ie, reward) from the contrast of positive feedback $>$ negative feedback for each individual. Individual contrast images were then used in second-level random effects models accounting for scan-to-scan and participant-to-participant variability to determine mean condition-specific regional responses using one-sample $t$-tests thresholded at $p<0.05$, FWE-corrected, and $\geqslant 10$ contiguous voxels. Our VS region of interest was constructed using the Talairach Daemon option of the WFU PickAtlas Tool, version 1.04 (Wake Forest University School of Medicine, Winston-Salem, NC). Two spheres of $10 \mathrm{~mm}$ radius were created around MNI coordinates $x= \pm 12, y=12$, and $z=-10$ to encompass the VS in the right and left hemisphere, respectively.

\section{Variance Analyses}

To compute the relative variance explained by the cumulative genetic profile score and each individual 
genotype, parameter estimates from VS clusters exhibiting a main effect of task were extracted using the VOI tool in SPM8 and entered into linear and stepwise regression models in SPSS (PASW Statistics 18; SPSS, Chicago, IL). Importantly, by extracting VS reactivity values from the entire functional clusters activated by our fMRI paradigm rather than clusters specifically correlated with our independent variables of interest, we precluded the possibility of any regression coefficient inflation that may result from capitalizing on the same data twice (Viviani, 2010). We have successfully used this more conservative analytic strategy in recent studies (Carre et al, in press; Hyde et al, 2010). Consistent with the standards of genetic association studies (eg, Dahlman et al, 2002) we applied a Bonferroni-like adjustment to our significance level to reflect the total number of regressions conducted (12 regressions total: 5 individual loci and 1 profile score conducted independently for the left and right VS) resulting in a threshold of $p \leqslant 0.004$ (ie, 0.05/12). Cook's distance values were computed for all observations and all regression models, and no single data points were identified that biased the overall models (ie, Cook's distance $<0.195$ for all data points) (Cook and Weisber, 1982).

On the basis of evidence for significant gender differences in reward-related VS reactivity in previous studies (Spreckelmeyer et al, 2009) and in our current sample (males > females; $t(67)=2.55, p=0.013)$ as well as an observed gender association with the profile scores (males $>$ females; $t(67)=2.12, p=0.038$ ), all our analyses were conducted with and without gender as a covariate. Thus, when gender was controlled for, the amount of variance explained by the genetic profile scores or individual loci was computed as the change in $R^{2}$ resulting from the addition of the genetic variables to a hierarchical regression model already containing gender as a predictor of VS reactivity.

\section{RESULTS}

In the current sample, there was a significant main effect of task (ie, positive feedback $>$ negative feedback) in a large VS cluster in the right hemisphere $(x=14, y=12, z=-8$,
$T=5.13, K_{E}=118, p=0.00028$; Figure 1a). There was also a main effect of task in a smaller cluster within the left VS $\left(x=-16, \quad y=6, \quad z=-8, \quad T=5.64, \quad K_{E}=96\right.$, $p=0.000043$; Supplementary Figure S1).

Consistent with our hypothesis, individual multilocus genetic profile scores for relatively increased DA signaling predicted higher reward-related reactivity in the right VS at our corrected threshold $(\hat{\beta}=0.342, p=0.0038)$. Moreover, the profile scores accounted for $10.9 \%$ of all variability within this VS cluster $\left(\Delta R^{2}=0.109\right.$; Figure $\left.1 \mathrm{~b}\right)$ above and beyond the effects of gender, which accounted for $4.2 \%$ of all residual cluster-level variability $(\hat{\beta}=-0.212$; $\left.\Delta R^{2}=0.042, p=0.067\right)$. When not explicitly controlling for gender, the DA profile scores predicted even greater variability in reward-related VS reactivity: $14.3 \%(\hat{\beta}=0.395$; Adj. $\left.R^{2}=0.143, p=0.001\right)$. In contrast, none of the individual loci predicted significant variability in rewardrelated VS reactivity with only one (DRD2 -141C Ins/Del) having a marginally significant effect at an uncorrected threshold of $p \leqslant 0.05$ (Table 2). Controlling for gender did not affect any of the associations between VS reactivity and individual loci.

Intriguingly, although none of the individual loci with the exception of DRD2 -141C Ins/Del accounted for significant variability in VS reactivity at an uncorrected threshold, the genetic profile scores still accounted for $6.3 \%$ of VS reactivity $\left(\hat{\beta}=0.298 ; \Delta R^{2}=0.063, p=0.026\right)$ above and

Table 2 Effects of Individual DA Loci on Reward-Related VS Reactivity

\begin{tabular}{|c|c|c|c|}
\hline Genotype & $\Delta R^{2}$ & $\hat{\boldsymbol{\beta}}$ & $p$ \\
\hline DRD2 I4IC Ins/Del & 0.052 & 0.241 & 0.050 \\
\hline DATI VNTR & 0.037 & 0.192 & 0.100 \\
\hline DRD4 VNTR & 0.015 & 0.123 & 0.295 \\
\hline COMT Val ${ }^{158} \mathrm{Met}$ & 0.015 & 0.123 & 0.298 \\
\hline DRD2 Taq IA & 0.002 & 0.045 & 0.713 \\
\hline
\end{tabular}

Critically, and as noted in the main text, none of these individual loci accounted for significant variability in VS reactivity when appropriately controlling for multiple comparisons (ie, $p \leqslant 0.004$ ).

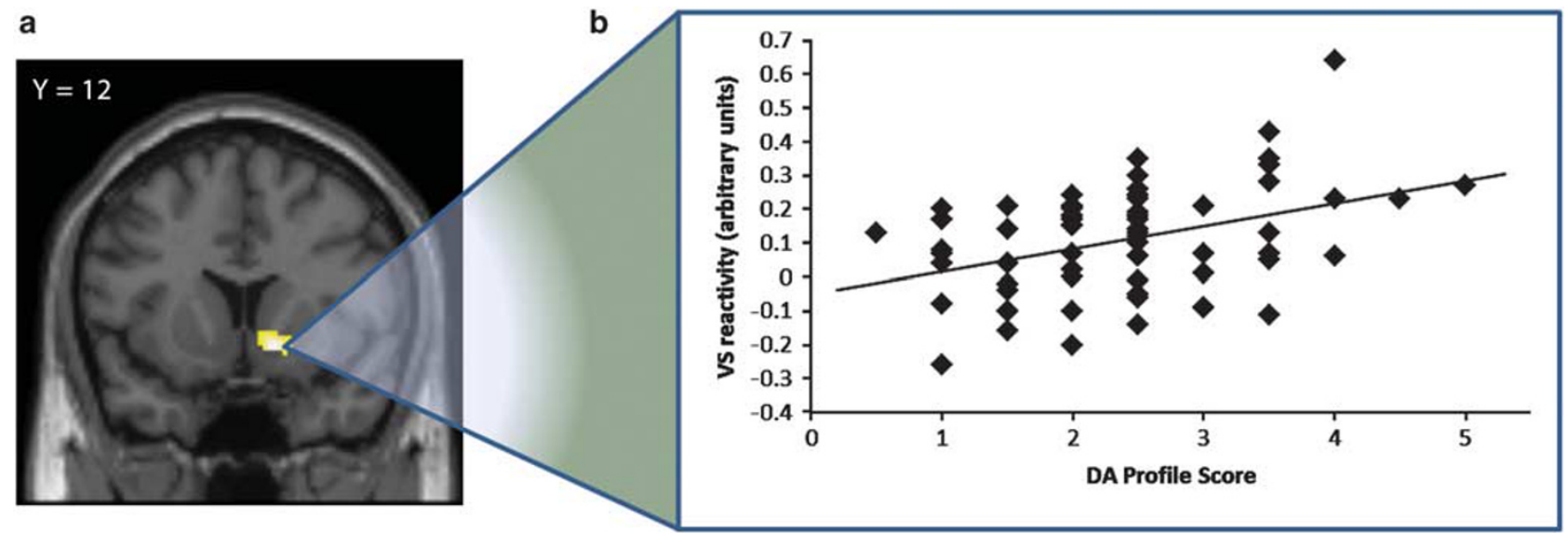

Figure I Multilocus genetic profile scores for DA signaling predict reward-related VS reactivity. (a) Our $\mathrm{PMRI}$ task produced significant activation in a large right VS cluster $\left(x=14, y=12, z=-8, T=5.13, K_{E}=1 \mid 8, p=0.00028\right)$. (b) Individual profile scores accounted for $10.9 \%$ of the variability within the VS activation cluster above and beyond the effects of gender $\left(\Delta R^{2}=0.109\right)$. 
beyond the effects of gender and DRD2 -141C Ins/Del. When gender was not used as a covariate, cumulative DA profile score accounted for $7.3 \%\left(\hat{\beta}=0.319 ; \Delta R^{2}=0.073\right.$, $p=0.019)$ of the variance above and beyond that accounted for by DRD2 -141C Ins/Del. The above results show the utility of multilocus genetic profiles in capturing the cumulative impact of polymorphisms, whose individual effects may be overlooked even at uncorrected statistical thresholds. Importantly, the simultaneous consideration of all predictors in the above regression models did not pose significant multicollinearity concerns (tolerance $>0.680$, variance inflation factor $<1.469$ ).

In contrast to the patterns observed in the right hemisphere, reward-related reactivity in the left VS was not significantly associated with the profile scores or any individual polymorphism at either the corrected or uncorrected statistical thresholds. Controlling for gender did not affect any of these associations.

\section{DISCUSSION}

In this study, we demonstrate that a multilocus genetic profile score representing the cumulative impact of five functional polymorphic loci on DA signaling predicts $10.9 \%$ of the inter-individual variability in reward-related VS reactivity. In contrast, none of the individual loci predict significant variability in VS reactivity. Thus, we provide novel evidence for the utility of biologically founded multilocus genetic profiles in mapping individual differences in brain function by demonstrating that simultaneous consideration of multiple functional loci accounts for greater variability than single loci considered independently. This finding demonstrates that, given sufficient a priori rationale for the consideration of specific functional polymorphic loci, a multilocus profiling approach might capture the cumulative impact of polymorphisms whose individual effects may otherwise go undetected in small samples. That the genetic profile scores in the current sample accounted for a significant proportion of variability in a relatively small sample further underscores the potential for this novel biological profiling approach to accurately predict patterns of brain function at the individual level.

Although we selected the polymorphisms investigated herein based on their previous association with DA signaling and/or VS reactivity, the precise molecular mechanisms through which each locus contributes to variability in reward-related brain function are still incompletely understood. Although the DAT1 9-repeat allele and the COMT ${ }^{158} \mathrm{Met}$ allele are linked to reduced DA synaptic clearance (Heinz et al, 2000) and enzymatic degradation (Chen et al, 2004), respectively, less is known about the direct effects of the $D R D 2$ and $D R D 4$ polymorphisms on DA neurotransmission and subsequent VS reactivity. The DRD4 7-repeat allele has been linked to reduced postsynaptic inhibition mediated by a decreased number of D4 receptors (Asghari et al, 1995). Relatedly, the DRD2 -141C Del allele has been associated with reductions in the expression of the D2 receptor, which typically acts to inhibit DA signaling pre- or postsynaptically (Arinami et al, 1997). Intriguingly, while previous research has linked the DRD2
Taq1A $\mathrm{T}$ (A1) allele to similarly reduced D2 receptor binding (Jonsson et al, 1999; Pohjalainen et al, 1998), studies investigating the effect of the polymorphism on regional blood flow and glucose metabolism have reported decreased striatal reactivity in $\mathrm{T}$ (A1) allele homozygotes relative to C (A2) allele carriers (Noble et al, 1997; Stice et $a l, 2008)$. As our VS reactivity phenotype is more closely related to the neuroimaging measures employed in the latter studies, we chose to code the $\mathrm{C}$ allele as the relatively 'high' DA allele. It is important to note, however, that the decreased D2 receptor density and the reduced glucose metabolism associated with the $\mathrm{T}$ allele need not be mutually exclusive. Given the diverse distribution of D2 receptors on multiple neuronal subtypes (Beaulieu and Gainetdinov, 2011), it is conceivable that reductions in D2 receptors associated with the $\mathrm{T}$ allele may be specific to a sub-population of D2 heteroreceptors located on GABAergic interneurons, which modulate striatal function through inhibition of glutamatergic medium spiny neurons. Thus, the $\mathrm{T}$ allele may result in reduced $\mathrm{DA}$-mediated inhibition of GABAergic interneurons leading to greater inhibition of excitatory medium spiny neurons and, ultimately, reduced VS reactivity measured with fMRI. Given the limitations of currently available neuroimaging methodologies, future studies incorporating non-human animal models will be required to determine the effects of each polymorphism on the cellular and systems levels with greater precision.

We previously reported that the 9-repeat allele of DAT1, the 7-repeat allele of DRD4, and the deletion allele of DRD2 -141C Ins/Del are all significantly associated with relatively increased VS reactivity in a sample that partially overlaps with our current study (Forbes et al, 2009). However, the previous sample was racially heterogeneous, as it included approximately $10 \%$ non-Caucasians distributed equally across all genotype groups. More importantly, in our previous report we used a less conservative approach whereby we only quantified VS reactivity as a function of genotype within functional clusters selected on the basis of their correlation with each polymorphic locus, rather than the entire functional cluster activated by our fMRI paradigm. Moreover, we did not apply statistical thresholds that properly accounted for multiple comparisons reflecting the number of individual genotypes tested. Importantly, we replicated the associations between VS reactivity and all three loci (ie, DAT1, DRD2 -141C Ins/Del, and DRD4 VNTR) when applying more liberal statistical thresholds consistent with our previous report (Supplementary Table S1).

In addition to the three polymorphisms investigated in our previous report, the cumulative genetic profile scores used in the current analysis also incorporated DRD2 Taq1A and COMT Val ${ }^{158}$ Met. Previously, we did not find a main effect of COMT $\mathrm{Val}^{158}$ Met on reward-related VS reactivity (Forbes et al, 2009). However, other imaging genetics studies have reported significant associations between the ${ }^{158}$ Met allele and increased VS reactivity (Dreher et al, 2009; Schmack et al, 2008; Yacubian et al, 2007). Thus, while COMT Val ${ }^{158}$ Met did not by itself predict significant variability in VS reactivity in either our previous (Forbes et al, 2009) or current analysis, it did significantly contribute to the predicted utility of the cumulative profile scores. Removal of the COMT Val ${ }^{158}$ Met genotype from the 
profile score resulted in nonsignificant effects using our corrected threshold.

A possible limitation to our biological profiling approach is the assumption that the selected polymorphisms act additively, as opposed to interactively, to influence DA signaling. Importantly, however, our previous investigation of three polymorphisms considered herein (ie, DAT1, DRD4 VNTR, and DRD2 -141C Ins/Del) did not find any two- or three-way interactions among these polymorphisms in predicting VS reactivity (Forbes et al, 2009). Unlike Yacubian et al (2007) and Dreher et al (2009), we also did not find a COMT Val ${ }^{158}$ Met-by-DAT1 interaction in the current sample ( $p=0.757)$. By assigning a score of ' 1 ' to 'high' DA alleles at all loci, we also assumed all loci had an equal impact on VS reactivity. We believe a more sophisticated approach to compiling genetic profiles is warranted whereby potential multiplicative relationships are taken into account and polymorphisms are weighted according to predicted effect size. However, given the relatively small sample size $(N=69)$ and insufficient knowledge regarding potential interactions among the targeted polymorphisms and the relative magnitude of their impact on VS reactivity (but see Yacubian et al, 2007 and Dreher et al, 2009), we regard the current investigation as a useful starting point for compiling informative multilocus genetic profile scores. Future studies replicating the current findings would lend additional credibility to this approach.

The DA profile we compiled in this study accounted for significant variability in reward-related reactivity of the right but not the left VS. Such asymmetrical findings are not uncommon in imaging genetics research in general (Hariri et al, 2002; Meyer-Lindenberg et al, 2006) or studies focusing on the VS specifically (Jocham et al, 2009; Yacubian et al, 2007). Although a number of studies have reported asymmetries in monoaminergic modulation of cortical and subcortical circuits (Besson and Louilot, 1995; Merali et al, 2004; Sullivan and Dufresne, 2006; Young and Williams, 2010), the biological mechanisms mediating such lateralized effects, particularly in the VS, are difficult to ascertain on the basis of the existing literature. It is possible that our specific task differentially recruits the right VS and, subsequently, results in greater DA modulation of reactivity in this hemisphere, which is reflected in the right-lateralized significant associations. Consistent with this suggestion, we have previously found right-hemisphere specific correlations between reward-related VS reactivity and variability in behavioral measures of impulsivity (Forbes et al, 2009; Hariri et al, 2006). Future research incorporating this genetic profile within a multimodal neuroimaging strategy (Fisher et al, 2009; Fisher et al, 2006; Kienast et al, 2008) whereby fMRI is used to measure reward-related VS reactivity and PET is used to measure DA release within the same sample could shed light on the nature of these functional asymmetries.

Although this proof-of-principle study focused on a single neural target and modeled the additive effects of multiple functional loci through a single genetic profile, future research can refine genetic profile scores by assigning differential weights to loci of potentially different effect sizes and consider functional interactions among loci within a profile as well as between profiles for different pathways. The extension of genetic profiling in this manner, particularly in larger samples, offers the opportunity to generate increasingly complete information regarding variability in behaviorally relevant brain function and related gene-environment interactions.

\section{ACKNOWLEDGEMENTS}

This work was supported by National Institutes of Health (NIH) Grants HL040962 to SBM and MH072837 to ARH. We thank Adam Gorka and Janet Lower for assistance with data collection and analyses.

\section{DISCLOSURE}

The authors declare no conflict of interest.

\section{REFERENCES}

Arinami T, Gao M, Hamaguchi H, Toru M (1997). A functional polymorphism in the promoter region of the dopamine D2 receptor gene is associated with schizophrenia. Hum Mol Genet 6: $577-582$.

Asghari V, Sanyal S, Buchwaldt S, Paterson A, Jovanovic V, Van Tol HH (1995). Modulation of intracellular cyclic AMP levels by different human dopamine D4 receptor variants. J Neurochem 65: 1157-1165.

Bakker PR, van Harten PN, van Os J (2008). Antipsychotic-induced tardive dyskinesia and polymorphic variations in COMT, DRD2, CYP1A2 and MnSOD genes: a meta-analysis of pharmacogenetic interactions. Mol Psychiatry 13: 544-556.

Beaulieu JM, Gainetdinov RR (2011). The physiology, signaling, and pharmacology of dopamine receptors. Pharmacol Rev 63: $182-217$.

Besson C, Louilot A (1995). Asymmetrical involvement of mesolimbic dopaminergic neurons in affective perception. Neuroscience 68: 963-968.

Buckholtz JW, Sust S, Tan HY, Mattay VS, Straub RE, MeyerLindenberg A et al (2007). fMRI evidence for functional epistasis between COMT and RGS4. Mol Psychiatry 12: 893-895, 885.

Buckholtz JW, Treadway MT, Cowan RL, Woodward ND, Benning $\mathrm{SD}$, Li R et al (2010a). Mesolimbic dopamine reward system hypersensitivity in individuals with psychopathic traits. Nat Neurosci 13: 419-421.

Buckholtz JW, Treadway MT, Cowan RL, Woodward ND, Li R, Ansari MS et al (2010b). Dopaminergic network differences in human impulsivity. Science 329: 532.

Carre JM, Fisher PM, Manuck SB, Hariri AR. Interaction between trait anxiety and trait anger predict amygdala reactivity to angry facial expressions in men but not women. Soc Cogn Affect Neurosci (in press).

Caspi A, Moffitt TE (2006). Gene-environment interactions in psychiatry: joining forces with neuroscience. Nat Rev Neurosci 7: 583-590.

Chen J, Lipska BK, Halim N, Ma QD, Matsumoto M, Melhem S et al (2004). Functional analysis of genetic variation in catechol-Omethyltransferase (COMT): effects on mRNA, protein, and enzyme activity in postmortem human brain. Am J Hum Genet 75: 807-821.

Cook R, Weisber S (1982). Residuals and Influence in Regression. Chapman \& Hall: New York.

Dahlman I, Eaves IA, Kosoy R, Morrison VA, Heward J, Gough SC et al (2002). Parameters for reliable results in genetic association studies in common disease. Nat Genet 30: 149-150.

Dalley JW, Fryer TD, Brichard L, Robinson ES, Theobald DE, Laane K et al (2007). Nucleus accumbens D2/3 receptors predict 
trait impulsivity and cocaine reinforcement. Science 315: 1267-1270.

Derringer J, Krueger RF, Dick DM, Saccone S, Grucza RA, Agrawal A et al (2010). Predicting sensation seeking from dopamine genes. A candidate-system approach. Psychol Sci 21: 1282-1290.

Dillon DG, Bogdan R, Fagerness J, Holmes AJ, Perlis RH, Pizzagalli DA (2010). Variation in TREK1 gene linked to depressionresistant phenotype is associated with potentiated neural responses to rewards in humans. Hum Brain Mapp 31: 210-221.

Dreher JC, Kohn P, Kolachana B, Weinberger DR, Berman KF (2009). Variation in dopamine genes influences responsivity of the human reward system. Proc Natl Acad Sci USA 106: 617-622.

Egan MF, Goldberg TE, Kolachana BS, Callicott JH, Mazzanti CM, Straub RE et al (2001). Effect of COMT Val108/158 Met genotype on frontal lobe function and risk for schizophrenia. Proc Natl Acad Sci USA 98: 6917-6922.

First MB, Spitzer RL, Gibbon M, Williams JBM (1996). Structured Clinical Interview for DSM-IV Axis I Disorders, Research Version, Non-Patient Edition. New York State Psychiatric Institute, Biometrics Research Department: New York.

Fisher PM, Meltzer CC, Price JC, Coleman RL, Ziolko SK, Becker C et al (2009). Medial prefrontal cortex 5-HT(2A) density is correlated with amygdala reactivity, response habituation, and functional coupling. Cereb Cortex 19: 2499-2507.

Fisher PM, Meltzer CC, Ziolko SK, Price JC, Moses-Kolko EL, Berga SL et al (2006). Capacity for 5-HT1A-mediated autoregulation predicts amygdala reactivity. Nat Neurosci 9: 1362-1363.

Forbes EE, Brown SM, Kimak M, Ferrell RE, Manuck SB, Hariri AR (2009). Genetic variation in components of dopamine neurotransmission impacts ventral striatal reactivity associated with impulsivity. Mol Psychiatry 14: 60-70.

Gan JO, Walton ME, Phillips PE (2010). Dissociable cost and benefit encoding of future rewards by mesolimbic dopamine. Nat Neurosci 13: 25-27.

Gianaros PJ, Manuck SB, Sheu LK, Kuan DC, Votruba-Drzal E, Craig AE et al (2010). Parental education predicts corticostriatal functionality in adulthood. Cereb Cortex 21: 896-910.

Hariri AR (2009). The neurobiology of individual differences in complex behavioral traits. Annu Rev Neurosci 32: 225-247.

Hariri AR, Brown SM, Williamson DE, Flory JD, de Wit H, Manuck SB (2006). Preference for immediate over delayed rewards is associated with magnitude of ventral striatal activity. J Neurosci 26: 13213-13217.

Hariri AR, Gorka A, Hyde LW, Kimak M, Halder I, Ducci F et al (2009). Divergent effects of genetic variation in endocannabinoid signaling on human threat- and reward-related brain function. Biol Psychiatry 66: 9-16.

Hariri AR, Mattay VS, Tessitore A, Kolachana B, Fera F, Goldman D et al (2002). Serotonin transporter genetic variation and the response of the human amygdala. Science 297: 400-403.

Heinz A, Goldman D, Jones DW, Palmour R, Hommer D, Gorey JG et al (2000). Genotype influences in vivo dopamine transporter availability in human striatum. Neuropsychopharmacology 22: 133-139.

Hyde LW, Gorka A, Manuck SB, Hariri AR (2010). Perceived social support moderates the link between threat-related amygdala reactivity and trait anxiety. Neuropsychologia 49: 651-656.

Ising $M$, Lucae $S$, Binder EB, Bettecken $T$, Uhr M, Ripke $S$ et al (2009). A genomewide association study points to multiple loci that predict antidepressant drug treatment outcome in depression. Arch Gen Psychiatry 66: 966-975.

Jocham G, Klein TA, Neumann J, von Cramon DY, Reuter M, Ullsperger M (2009). Dopamine DRD2 polymorphism alters reversal learning and associated neural activity. J Neurosci 29: 3695-3704.

Jonsson EG, Nothen MM, Grunhage F, Farde L, Nakashima Y, Propping $\mathrm{P}$ et al (1999). Polymorphisms in the dopamine D2 receptor gene and their relationships to striatal dopamine receptor density of healthy volunteers. Mol Psychiatry 4: 290-296.

Kienast T, Hariri AR, Schlagenhauf F, Wrase J, Sterzer P, Buchholz HG et al (2008). Dopamine in amygdala gates limbic processing of aversive stimuli in humans. Nat Neurosci 11: 1381-1382.

Kwon JS, Kim E, Kang DH, Choi JS, Yu KS, Jang IJ et al (2008). Taq1A polymorphism in the dopamine D2 receptor gene as a predictor of clinical response to aripiprazole. Eur Neuropsychopharmacol 18: 897-907.

Merali Z, McIntosh J, Anisman H (2004). Anticipatory cues differentially provoke in vivo peptidergic and monoaminergic release at the medial prefrontal cortex. Neuropsychopharmacology 29: 1409-1418.

Meyer-Lindenberg A, Buckholtz JW, Kolachana B, A RH, Pezawas L, Blasi G et al (2006). Neural mechanisms of genetic risk for impulsivity and violence in humans. Proc Natl Acad Sci USA 103: 6269-6274.

Nicodemus KK, Callicott JH, Higier RG, Luna A, Nixon DC, Lipska BK et al (2010). Evidence of statistical epistasis between DISC1, CIT and NDEL1 impacting risk for schizophrenia: biological validation with functional neuroimaging. Hum Genet 127: 441-452.

Noble EP, Blum K, Ritchie T, Montgomery A, Sheridan PJ (1991). Allelic association of the D2 dopamine receptor gene with receptor-binding characteristics in alcoholism. Arch Gen Psychiatry 48: 648-654.

Noble EP, Gottschalk LA, Fallon JH, Ritchie TL, Wu JC (1997). D2 dopamine receptor polymorphism and brain regional glucose metabolism. Am J Med Genet 74: 162-166.

Pezawas L, Meyer-Lindenberg A, Goldman AL, Verchinski BA, Chen G, Kolachana BS et al (2008). Evidence of biologic epistasis between BDNF and SLC6A4 and implications for depression. Mol Psychiatry 13: 709-716.

Plomin R, Haworth CM, Davis OS (2009). Common disorders are quantitative traits. Nat Rev Genet 10: 872-878.

Pohjalainen T, Rinne JO, Nagren K, Lehikoinen P, Anttila K, Syvalahti EK et al (1998). The A1 allele of the human D2 dopamine receptor gene predicts low D2 receptor availability in healthy volunteers. Mol Psychiatry 3: 256-260.

Potkin SG, Guffanti G, Lakatos A, Turner JA, Kruggel F, Fallon JH et al (2009). Hippocampal atrophy as a quantitative trait in a genome-wide association study identifying novel susceptibility genes for Alzheimer's disease. PLoS One 4: e6501.

Schmack K, Schlagenhauf F, Sterzer P, Wrase J, Beck A, Dembler T et al (2008). Catechol-O-methyltransferase val158met genotype influences neural processing of reward anticipation. Neuroimage 42: 1631-1638.

Sesack SR, Grace AA (2010). Cortico-basal ganglia reward network: microcircuitry. Neuropsychopharmacology 35: 27-47.

Seshadri S, DeStefano AL, Au R, Massaro JM, Beiser AS, KellyHayes $M$ et al (2007). Genetic correlates of brain aging on MRI and cognitive test measures: a genome-wide association and linkage analysis in the Framingham Study. BMC Med Genet 8(Suppl 1): S15.

Spreckelmeyer KN, Krach S, Kohls G, Rademacher L, Irmak A, Konrad K et al (2009). Anticipation of monetary and social reward differently activates mesolimbic brain structures in men and women. Soc Cogn Affect Neurosci 4: 158-165.

Stice E, Spoor S, Bohon C, Small DM (2008). Relation between obesity and blunted striatal response to food is moderated by TaqIA A1 allele. Science 322: 449-452.

Sullivan RM, Dufresne MM (2006). Mesocortical dopamine and HPA axis regulation: role of laterality and early environment. Brain Res 1076: 49-59.

Tanaka SC, Doya K, Okada G, Ueda K, Okamoto Y, Yamawaki S (2004). Prediction of immediate and future rewards differentially recruits cortico-basal ganglia loops. Nat Neurosci 7: 887-893. 
van de Giessen E, de Win MM, Tanck MW, van den Brink W, Baas F, Booij J (2009). Striatal dopamine transporter availability associated with polymorphisms in the dopamine transporter gene SLC6A3. J Nucl Med 50: 45-52.

VanNess SH, Owens MJ, Kilts CD (2005). The variable number of tandem repeats element in DAT1 regulates in vitro dopamine transporter density. BMC Genet 6: 55.

Viviani R (2010). Unbiased ROI selection in neuroimaging studies of individual differences. Neuroimage 50: 184-189.
Wang E, Ding YC, Flodman P, Kidd JR, Kidd KK, Grady DL et al (2004). The genetic architecture of selection at the human dopamine receptor D4 (DRD4) gene locus. Am J Hum Genet 74: 931-944.

Yacubian J, Sommer T, Schroeder K, Glascher J, Kalisch R, Leuenberger B et al (2007). Gene-gene interaction associated with neural reward sensitivity. Proc Natl Acad Sci USA 104: 8125-8130.

Young EJ, Williams CL (2010). Valence dependent asymmetric release of norepinephrine in the basolateral amygdala. Behav Neurosci 124: 633-644.

Supplementary Information accompanies the paper on the Neuropsychopharmacology website (http://www.nature.com/npp) 